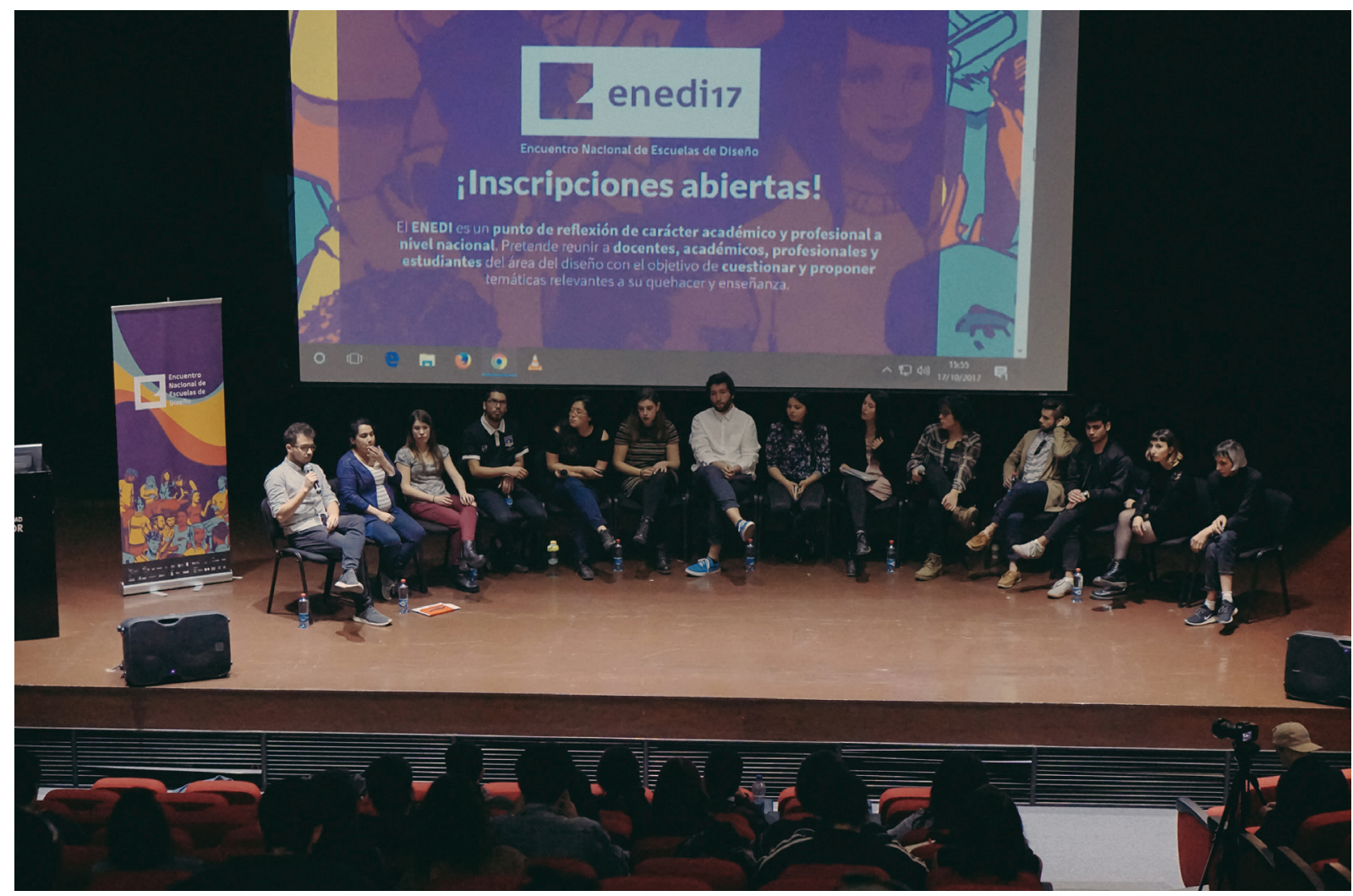

RITA PAZ TORRES

ESCUELA DE DISEÑO

FACULTAD DE ARQUITECTURA Y URBANISMO UNIVERSIDAD DE CHILE

SANTIAGO, CHILE

RITAPAZTORRES@GMAIL.COM

Cómo citar: Torres, R. P. (2017). Encuentro Nacional de Escuelas de Diseño 2017 (ENEDI). RChD: creación y pensamiento, 2(3), 1-7. DOI: $10.5354 / 0719-837 X .2017 .47826$

1. Luego de su relanzamiento, en el año 2010 ENEDI se trasladó fuera de la capital, siendo organizada por la Universidad Católica de Temuco. Asimismo, y debido a la contingencia política y social de las movilizaciones estudiantiles del 2011, se realiza el Encuentro Movilizado de Estudiantes de Diseño (EMEDI) en ciudad de La Serena.

\section{Encuentro Nacional de Escuelas de Diseño 2017 (ENEDI) | ENEDI 2017}

\section{Rita Paz Torres, Directora de Contenido ENEDI 2017}

Entre el pasado 17 y 20 de octubre del 2017, se realizó en Santiago el Encuentro Nacional de Escuelas de Diseño (ENEDI), invitando a discutir y problematizar discursos que existen en torno a la enseñanza de dicha profesión. Este Encuentro, desde su relanzamiento en el año 2009 en la Facultad de Arquitectura y Urbanismo de la Universidad de Chile, ha sido desarrollado intermitentemente por grupos de estudiantes y docentes de diferentes universidades ${ }^{1}$ con excepción de su penúltima versión 2014, la cual fue levantada de forma autogestionada por el mismo equipo organizador de esta versión. A diferencia de años anteriores, esta vez, ENEDI contó con el apoyo y financiamiento del Fondo Nacional para el Desarrollo Cultural y las Artes (FONDART), permitiendo ampliar el espectro de participantes en el evento y potenciar el impacto de la educación del diseño, consolidando al Encuentro a nivel país y proyectándose para próximas versiones futuras.

Esta quinta versión -organizada por el equipo de diseñadores Francisco Rojas, Augusto Causa, Valentina Oyarzún, Diego Salinas, Matías Aracena, Rita Torres y los asesores académicos del Departamento de Diseño de la Universidad de Chile, Simoné Malacchini y Eduardo Castillo- buscó levantar y democratizar espacios de discusión sobre la educación y el aporte público del quehacer del diseño y el impacto de la profesión en la sociedad. Mediante variadas activida- 


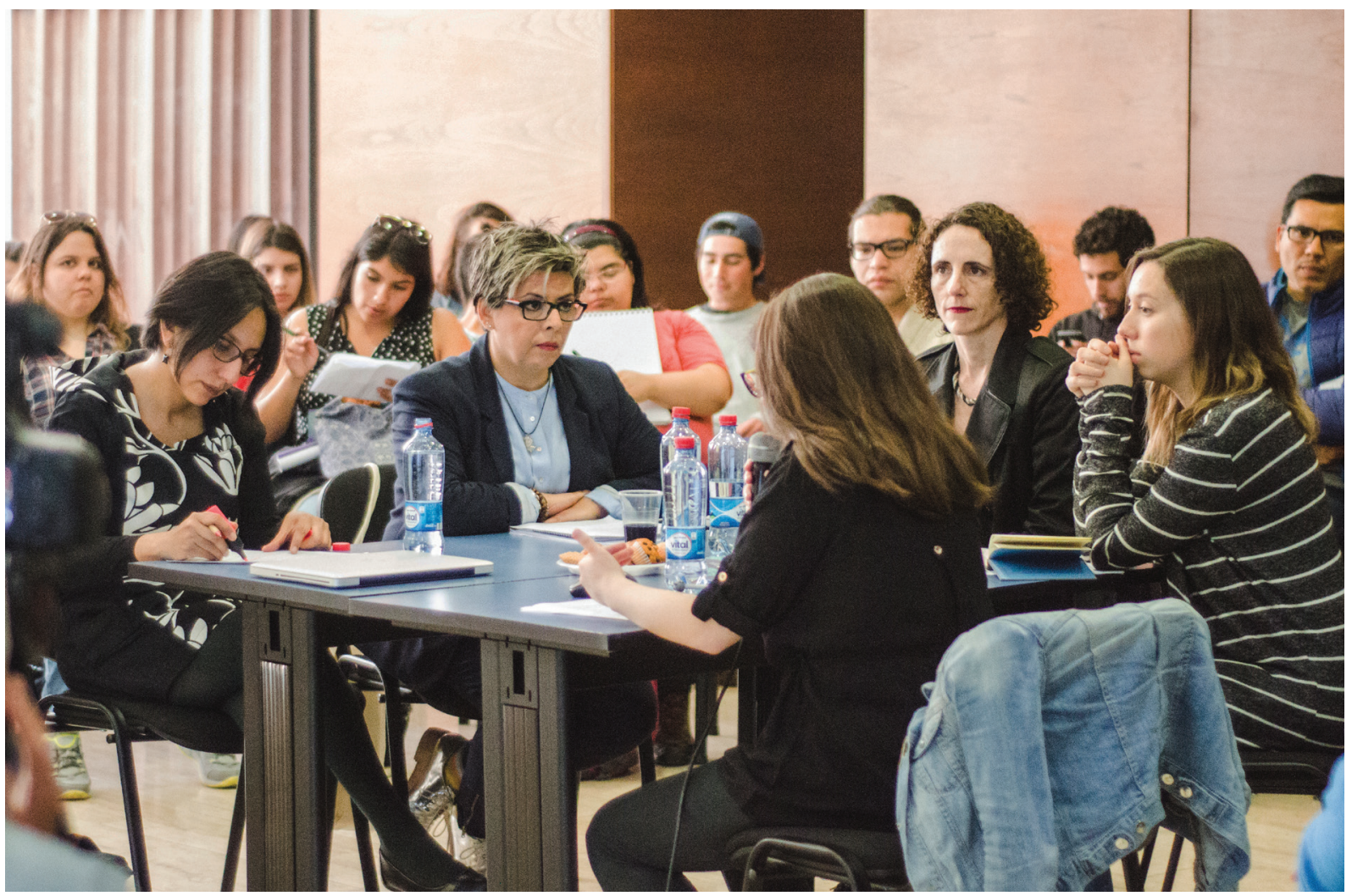

des, el debate y reflexión se llevó a cabo a través de los más diversos lineamientos, miradas y procesos que se desarrollan en la enseñanza del diseño, tales como la innovación, producción, creación e investigación a nivel país. ENEDI se encuentra inserto en el contexto actual de la educación, donde existe una gran cantidad de escuelas que ofertan la carrera de diseño a nivel nacional, además de contar con diversos espacios vinculados prioritariamente a exhibir la producción de esta área creativa. Esta realidad contrasta con las pocas instancias de debate abiertos y público, y que además se sostengan en el tiempo. Asimismo, las discusiones de índole educativa se realizan de forma interna y cerrada en las escuelas, por lo que resulta pertinente abrir la conversación para que las distintas visiones sobre la enseñanza en diseño puedan encontrarse y enriquecer el debate, estableciendo a esta práctica como una temática de interés público nacional.

Fueron cuatro días cargados de actividades, las cuales estuvieron concentradas geográficamente en el eje Alameda con Portugal, entre las dependencias de la Facultad de Arquitectura y Urbanismo de la Universidad de Chile y el Campus El Claustro de la Universidad Mayor, dos de las universidades patrocinadoras del Encuentro, además de la Universidad Católica de Chile. Entre los asistentes destacan importantes delegaciones de estudiantes provenientes de diversas regiones y universidades del país, como la Universidad del Bío-Bío, Universidad de Valparaíso, Universidad Católica de Temuco, y por supuesto, estudiantes, docentes y académicos de la Universidad Diego Portales, Universidad Tecnológica Metropolitana, Universidad del DesarroIlo, Universidad Adolfo Ibáñez, entre otras, además de las instituciones
Figura 1. Conversación abierta con los 14 estudiantes responsables de los póster de investigación seleccionados, con la moderación de Karen Hormazabal. Actividad realizada en el auditorio del Campus El Claustro de la Universidad Mayor.

Figura 2. Mesa Redonda de Investigación, realizada en el hall del auditorio del Campus El Claustro de la Universidad Mayor. De izquierda a derecha: Leslye Palacios, Pamela Gatica, Úrsula Bravo y Luciana Pastor. 


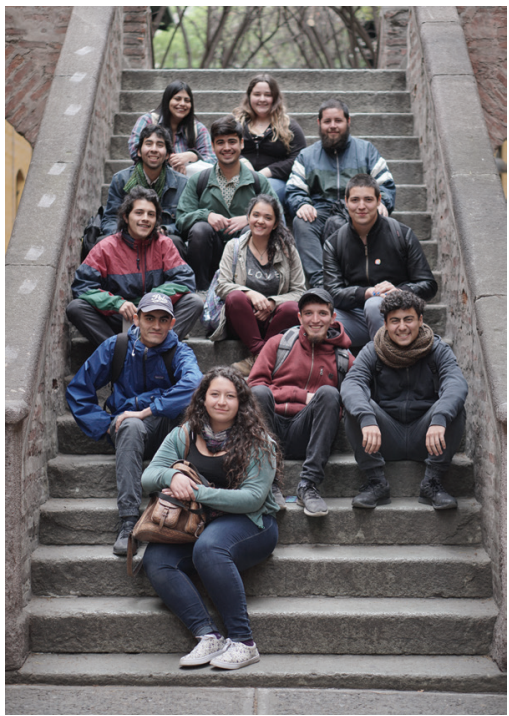

Figura 3. Representantes de los centros de estudiantes de diversas universidades e instituciones de Valparaíso, Temuco, Chillán, Concepción y Santiago, los cuales participaron en la actividad "Foro Estudiantil" en el Ágora de la Facultad de la Arquitectura y Urbanismo de la Universidad de Chile. Figura 4. Taller "Diseño de Interfaz Sonora" guiado por el Colectivo 22bits, realizado en la Facultad de la Arquitectura y Urbanismo de la Universidad de Chile.

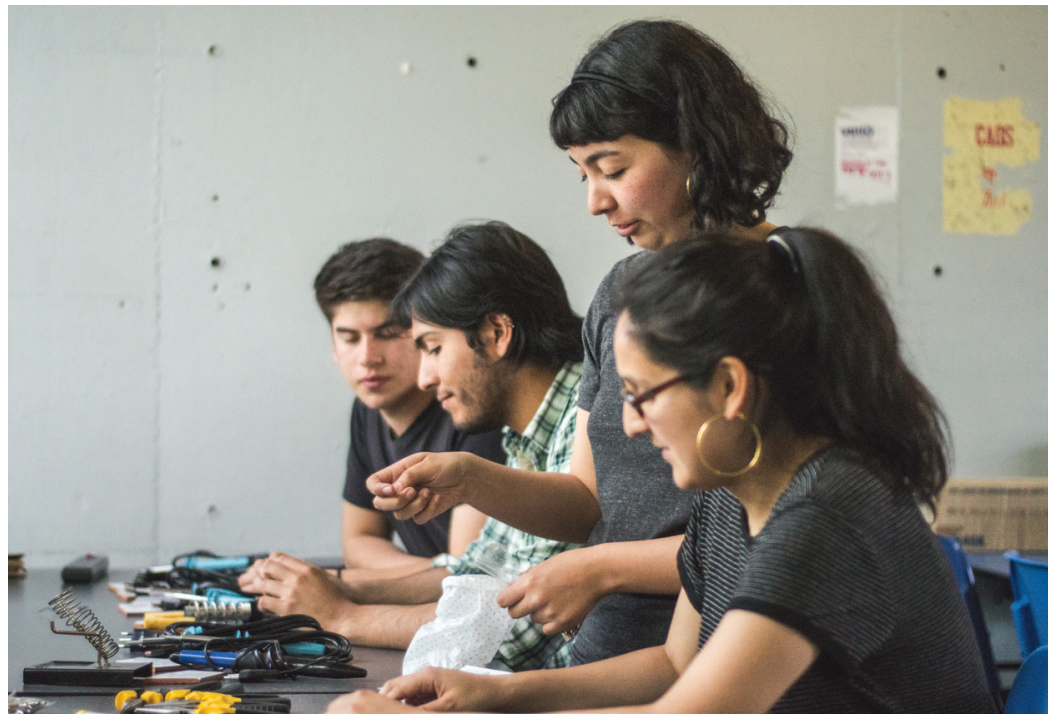

educativas patrocinadoras. También se hicieron parte de esta versión diversos espacios e instituciones como el Consejo Nacional de la Cultura y las Artes (CNCA), el Laboratorio de Gobierno, Chile Diseño, +D "Diseño para la Ciencia”, Stgo MakerSpace, IF, OpenD “Diseño para Todos”, entre otros. Con una asistencia de más de mil asistentes, este año se convocó a un público diverso, desde estudiantes, académicos, docentes, profesionales e incluso personas no relacionadas directamente con el diseño, de otras carreras, profesiones y oficios, los cuales participaron en más de 30 actividades gratuitas propuestas en el evento tales como: Mesas Redondas (innovación, creación, investigación y fabricación en diseño), Conversatorios y Talleres. También se utilizaron otros tipos de dinámicas, como IGNITE, en el Centro Cultural y Social El Corral, y las Charlas Autorales en el Espacio Nacional de Diseño (E.N.D.), junto con la exposición de Póster de investigación estudiantil y la Feria de Diseño Estampida en las Pasarelas Verdes San Borja, ampliando así, la puesta en circulación del conocimiento y producción del diseño.

\section{Dinamizar la discusión desde fuera de Santiago: ENEDI TOUR}

Conscientes que Santiago no es Chile y que no es posible abarcar otras miradas si no se abre la invitación, el equipo organizador de este año decidió gestionar como una estrategia de difusión del Encuentro el proyecto ENEDI TOUR, iniciativa que se origina con el objetivo de integrar a otras regiones del país a la reflexión, recogiendo miradas, experiencias, problemáticas y lineamientos lo más pluralista y diverso posible. Asimismo, se torna importante potenciar y gestionar instancias de conversación sobre el trabajo actual del diseño en diversos territorios, extendiendo la invitación a discutir de forma local las numerosas visiones del ejercicio profesional. Esta itinerancia se realizó entre los meses de junio y agosto, visitando la Universidad Santo Tomás en Valdivia, la Universidad Católica de Temuco, la Universidad del Bío Bío en sus sedes Chillán y Concepción, la Universidad Santo Tomás en Concepción y la Universidad de Valparaíso. Lo interesante y enriquecedor de este proyecto fue que todo lo conversado en las diferentes escuelas e instituciones educativas sirvió posteriormente, como insumo para plantear y guiar las conversaciones pensadas para cada mesa redonda, conversatorio, entre otros, durante el evento de octubre. La 




oportunidad de conocer y formar redes con diversos actores de la comunidad educativa del diseño de distintas partes de Chile, permitiendo poner en valor, de manera pública, las múltiples visiones de la práctica y enseñanza del diseño, visualizar las diferencias que existen entre las regiones y Santiago. Generar un llamado de atención en relación a cómo el centralismo afecta al desarrollo del diseño en regiones.

No es secreto que la mayoría de las oportunidades laborales y decisiones importantes en torno al diseño se concentran en gran medida en Santiago, especialmente desde la gestión y organización de actividades de promoción de este tales como seminarios, bienales, ferias, incluso el mismo ENEDI. A pesar de lo anterior, cerca de 150 representantes de diversas universidades de región llegaron al Encuentro a exponer, participar y reflexionar sobre cómo se están formando diseñadores en Chile, aportando inmensamente en las diferentes actividades a través de numerosas intervenciones y comentarios, lo que posibilitó estrechar lazos para futuras colaboraciones en un ambiente entusiasta y fraterno, proyectando múltiples posibilidades de seguir levantando instancias de conversación en torno al diseño y su enseñanza desde un mirada crítica y descentralizada.

\section{Producción reflexiva como insumo para nuevas discusiones: Publicación ENEDI}

Al plantear ENEDI como un espacio intrínsecamente relacionado con la práctica y enseñanza del diseño y cómo ésta puede llegar a ser una temática de interés público a nivel país, se torna sumamente relevante divulgar lo conversado y realizado en el Encuentro. Este ejercicio de producción reflexiva busca dar a conocer, convocar y seguir levantando instancias de diálogo, quedando como un antecedente a futuras discusiones relacionadas a la formación de diseñadores en Chile. Al querer observar y recordar las anteriores versiones de ENEDI, se torna problemático poder superar la barrera del tiempo en cuanto a constatar sobre qué se habló, conversó y discutió en cada versión, apoyándonos solamente en el registro oral de algunos de sus asistentes y participantes. Comprendiendo que la experiencia empírica es efímera, publicar se transforma en el soporte de memoria capaz de mediar con los


Figura 5. Charla Autoral a cargo del colectivo tipográfico W Type Foundry, realizada en el Espacio Nacional de Diseño (E.N.D.).

Figura 6. Segunda y última sesión de IGNITE en

El Corral Centro Cultural a través de una serie de charlas cortas sobre proyectos de diseño.

Figura 7. Conversatorio "Metodologías de diseño en espacios educativos", realizada en el auditorio de la Facultad de Arquitectura y Urbanismo de la Universidad de Chile. De izquierda a derecha: Fernanda Tapia, Roman Yosif, Kathya Morón y Jacob Bustamante. 
actuales y futuros diseñadores, posibilitando el estudio y análisis de aquellas experiencias, interacciones y diálogos realizados en este Encuentro 2017. Es así como la publicación está planteada desde la misma inquietud del grupo de trabajo y coordinación de este año, actuando desde una energía crítica y disidente en cuanto a formular más preguntas que respuestas o verdades. No busca aunar criterios ni llegar a consensos, sino albergar la diversidad de discursos, actores, espacios e instituciones relacionadas con el diseño en nuestro país, creyendo firmemente que todos y todas tienen algo que decir en torno a la formación de nuevos diseñadores. Publicar se vuelve menester para que exista un antecedente del diálogo, una especie de "fotografía" de las discusiones de diseño que se daban en Chile en el año 2017. En adición, toma gran importancia dejar registro de la discusión y las ideas planteadas, ya que es difícil plantear nuevas preguntas si no somos capaces ampliar el debate a quienes no fueron parte del encuentro, por temas espaciales o temporales, teniendo como gran meta que este material escrito se encuentre disponible en todas las escuelas e instituciones educativas a nivel nacional relacionadas al diseño, de forma impresa y digital, para su acceso libre y democrático.

\section{Proyecciones y carta de navegación hacia una mirada política del diseño}

Finalmente, este año se torna muy importante en relación a nuestro quehacer. A cincuenta años de la creación de los primeros cursos de diseño en el país en la Universidad de Chile, sede Valparaíso, y a unos meses del lanzamiento de la primera Política Nacional de Fomento al Diseño (2017-2022), ENEDI extiende la invitación a conversar sobre aquellos lineamientos y reflexiones que definen qué es lo hacemos cuando hacemos diseño, y cómo podemos ser un aporte a la sociedad, a través de una mirada sensible y creativa, partiendo desde la base que la educación es el eje más importante dentro de las políticas que impulsan el desarrollo de un país. En relación a lo anterior, para poder reflexionar sobre el estado actual de la enseñanza del diseño, es importante conocer y entender cómo se pretende enseñar y con qué objetivo se forman los profesionales de este quehacer. Entendiendo el impacto cada vez más claro que tiene el diseño en la economía, las cadenas de producción, la calidad de vida de los ciudadanos, los problemas sociales, las artes y las culturas, nuestra profesión resulta una arista que permite observar e incidir en diversos entornos, contextos y comunidades, un lenguaje potencialmente transformador, capaz de forjar múltiples imaginarios e identidades, el cual no debe ser dejado de lado a la hora de proyectar el futuro, lo que conlleva una gran responsabilidad en su ejercicio y enseñanza. Esta versión del Encuentro se enfocó en proponer espacios de conversación, colaboración y aprendizaje desde una mirada horizontal, crítica y pluralista, teniendo gran protagonismo el formato de las mesas redondas, siendo un éxito desde la diversidad de estudiantes, docentes, académicos y profesionales del área que convocaron a exponer tanto como a participar a través de comentarios y preguntas, las cuales retroalimentaron la discusión. Todo esto reafirmó la necesidad latente de seguir proyectando instancias de reflexión y encuentro en torno a nuestra disciplina a nivel nacional.

Finalmente, ENEDI es por sobre todas las cosas un punto de encuentro. Un llamado a detenerse, reunirse y abrir la discusión respecto a qué estamos produciendo, investigando, innovando, creando y con qué proyecciones se está y se quiere enseñar la práctica del diseño en Chile. El diseño se encuentra inserto en un territorio político que disputar y no es posible -o no sería recomendable- avanzar en el desarrollo de la profesión sin conocernos y colaborar entre nosotros. 




\section{Participantes ENEDI 2017}

Mesas Redondas

1. Innovación: Juan Quiroz, Gianncarlo Durán (Laboratorio de Gobierno), Natalia Oviedo (+D), Alejandro Pantoja (UDD). Moderador: Francisco Rojas (ENEDI).

2. Creación: Hugo Rivera-Scott (UCH), Carolina Pino (UAI), Juan Pablo Fuentes (E.N.D.), Paola Moreno (PUC). Moderador: Diego Salinas (ENEDI).

3. Investigación: Leslye Palacios (UCT), Pamela Gatica (UTEM), Luciana Pastor (UCH), Úrsula Bravo (UDD). Moderadora: Rita Torres (ENEDI).

4. Fabricación: Gabriela Pérez (Stgo MakerSpace), Rodrigo Dueñas ( $\mathrm{UCH}$ ), Fabiola López (D-IF), Franco Jonas, Camila Ríos (PUC). Moderador: Augusto Causa (ENEDI).

\section{Conversatorios}

1. Enseñanza v/s Ejercicio Profesional: Roberto Concha (Chile Diseño), Joel Espinoza (OpenD), Fabiola Prischtt, Andrés Villela. Moderador: Gonzalo Molina (OpenD).

2. Mallas Curriculares: Diana Henry (UCH), Izaul Parra (UBB), Matías Armijo. Moderador: Eduardo Castillo (UCH).

3. Educación No Formal: Gianncarlo Durán, Macarena Pola, Jacob Bustamante $(\mathrm{UCH})$. Moderadora: Simoné Malacchini (UCH)

4. Diseño y Medios: Roberto Osses (UCH), Michel Contreras, Patricia Peña (ICEI). Moderadora: Macarena Herrera (El Desconcierto)

5. Sustentabilidad en Diseño: Beatriz Venti, Pablo Zúñiga, Mónica Morales. Moderadora: Luna Mella (Centro de Estudiantes de Diseño UM)

6. Mujeres, diseño y formación: Sol Díaz, Anamias Ixaya, Paola Molina. Moderadora: Dai Liv Fuentes.

7. Diseño y accesibilidad: César Domínguez, Catalina Pérez, Patricio Gómez. Moderadora: Catalina Silva (Centro de Estudiantes de Diseño UM).
Figura 8. 6ta versión de la Feria de Diseño Estampida, espacio de difusión de proyectos independientes, realizada en las Pasarelas Verdes San Borja, junto al apoyo y colaboración del colectivo Estampida. 
8. Metodologías en diseño en espacios educativos: Fernanda Tapia, Roman Yosif (Laboratorio de Gobierno), Kathya Morón. Moderador: Jacob Bustamante.

Póster de Investigación

Moderadora: Karen Hormazábal (UTEM)

Diana Henry (UCH); Esperanza Jiménez (UDD); Maribel Sainz (UCH); Martina Citarella y Nicole Rosselot (UDP); Macarena Espina y Sofía Fuentes (UCH); Camille Descazeaux (UCH); Franco Jonas y Sebastian Lizama (UTEM); Nicolás Román y Margarita Talep (UDP); Pascuala Sylleros (UDD); Génesis Frías (UV); Camila Martin (UCH); Sebastián Serey, Luciana Toscanini y Milan Espinoza (UDP); Manuel Lara (UTEM); Lys Alves, Esteban Echeverría, María José Monroy y Natalia Trujillo (UCH).

\section{Talleristas}

Erik Ciravegna, Zinna Silva y Maite Nazarre (Applied Theatre in Design), Andrea Meza, Constanza Pavis y Rodrigo Morales (Libro Origami), Daniela Humeres (Creatividad y Experimentación Material), Felipe Cortez y Ricardo Vega (Visualización de Datos), Güiña-Imprenta Artesanal (Tipos Móviles Experimentales), Juan Carlos Silva (Interactivo en HTML5), Colectivo 22bits (Diseño de Interfaz Sonora), Pablo Madrid y Leo Calderón (Caligrafía con Brush Pen), Michel Contreras (Medios e Infografía), Claudio Navarro y Nelson Rodríguez (Lean Ux).

Charlas Autorales en E.N.D.

Minigolf Books; Convictus; Mestiza Joyería; y WFoundry.

Charla CNCA: "Introducción al diseño de proyectos de fondos concursables en Diseño", de Trinidad Guzmán y Rocío Pinto. 